\title{
Continuous quality improvement in nursing
}

\author{
Jennifer Hunt
}

Most people have an image of what nurses are and what they do. In most instances this reflects a combination of historical stereotypes and those induced by the media. At different periods and in different health care institutions nurses have done, and indeed still do, work which could be considered the province of many other staff: cleaners, porters, receptionists, technicians, managers, and doctors. Nursing - that is, the work nurses do - is in fact a complex and demanding activity. It involves assessing patients' nursing requirements; planning and implementing nursing care; carrying out medical orders; teaching staff (nursing and non-nursing) and patients' relatives; managing, organising, and coordinating services; and researching and evaluating the effectiveness of the nursing care provided. Nurses therefore need to be knowledgeable and skilled and, of course, caring. Nursing too is the patient care service in hospitals which is provided continuously 24 hours a day, seven days a week, 52 weeks of the year. Nurses therefore spend more time with their patients than other health care professionals, which provides them with a unique opportunity really to know and understand patients' needs and wishes. More importantly, research is showing that skilled nursing affects patient outcomes. Assuring the provision of good nursing care is therefore not a luxury but an essential component of effective patient care and of patient satisfaction.

During the past thirty years there have been two previous peaks of interest in quality improvement, the first in the late 1960 s and the second in the early 1980s. We now have a third and much more sustained period dating from, and stimulated by, the implementation of the Griffiths report ${ }^{1}$ and maintained by a continuing focus on the need for quality by government, management, professionals, and consumers. Unlike the previous initiatives, nurses are now part of a multidisciplinary effort, which means that the outcomes are likely to be more significant and more long lasting, particularly as health service organisations at all levels move into total quality management.

\section{Theory}

Although the nursing literature is large it does not show any coherent pattern, rather a great deal of duplication and reinventing of the wheel. Three main reasons account for this: firstly, the unstable, changing nature of definitions of quality; secondly, interest being intermittent; and, thirdly, interest often being

related to internal factors and pressures, such as shortages of nursing staff, and which fades when the problem is resolved or is superceded. As a result there has in the past been more talk and more theory than either action or implementation.

In their work relating to quality nurses have taken various approaches; these fall into four categories: (a) describing quality, (b) measuring quality, (c) comparing outcomes, or (d) any combination of these. The most recent developments, although incorporating these approaches, can be subdivided into generic audit and standard setting.

\section{Generic audit}

Unlike medicine, nursing in Britain at least, has frequently opted for developing a generic audit tool - that is, trying to get an overall measure of qualify for a ward, department, or hospital. Such measures include tools such as Monitor and Qualpacs. An additional dimension with Monitor, for example, is the link with staffing (both numbers and grade mix) and patient dependency, which gives it a strong management focus. The general nature of this approach limits the usefulness of these instruments, but, even so, the implementation of such a system has often proved helpful and led to further quality initiatives as long as nursing staff caring for patients were fully involved and they were not merely driven by management from the top down. One further problem is that evidence suggests that these different audit instruments give different results even when used in the same settings and with the same group of patients. In other words, they are measuring different aspects of quality or the same aspects but from different perspectives.

\section{Standard setting}

The idea of setting standards is well established. Standards range from very broad, almost philosophical, statements to very detailed protocols and procedures. Measuring the effect that the implementation of such standards has on patient care and patient outcomes has received less attention and of course is more difficult. In the United Kingdom a major development has been the setting up of the Royal College of Nursing dynamic standard setting system as part of its programme on standards of care, which has given nurses at ward level and in the community a flexible yet organised system that they can work with locally and which also has a nationwide network of users and national leadership. 
(1) To establish the direction and practices of the nursing quality assurance programme and to monitor and review its progress

(2) To approve all standards, policies, and protocols

(3) To approve action plans implemented to resolve problems

Box 1 Terms of reference of Nursing Quality Assurance Committee

Integrating theory with practice

What is happening to achieve integration of theory with practice and to ensure that standards affect nurses' behaviour and performance all day every day? Before 1989 several quality initiatives were under way within the special health authority - for example, several quality circles had been set up and a patient satisfaction questionnaire had been developed and was administered regularly. However, it became clear that these initiatives needed better coordination so the Nursing Quality Assurance Committee was set up to provide overall direction and leadership (box 1). Its membership includes all the key professional leaders in the special health authority as well as nurses engaged daily in quality activities (box 2). Meeting four times a year, the committee devolves the work down to the grass roots through three subgroups (box 2) which arrange and organise their own meetings. This structure is not seen as set in stone, rather it is an attempt to:

- Cover a wide range of quality assurance activities

- Involve nurses at all levels in quality assurance

- Provide opportunities for learning and practising different skills and techniques

- Enable nursing quality assurance activities to be coordinated and monitored.

All of the groups have developed an active programme. That of the standards group is the most well developed because the work on standard setting using the dynamic standard setting system had begun in 1990. Its achievements show how improvements can be made and maintained. The process consists of:

(1) Setting the standard

(2) Auditing current practice

(3) Implementing the standard (4) Reauditing practice and identifying
deficiencies
Chief nurse/director of quality

Directors of nursing services

Director of education

Quality assurance coordinator

Nurse practice advisers

Coordinators of subgroups (nursing audit, standards, and policies and procedures)

Box 2 Membership of Nursing Quality Assurance Committee

(5) Agreeing an appropriate action plan

(6) Reauditing perhaps six months later or so.

Already many topics have been addressed in this way - for example, management of postoperative pain in adult cardiothoracic and cardiovascular surgical patients and discharge planning for all inpatients. More are being added all the time. The advantage of this approach is that it really involves the nursing staff so that they "own" the process and the results. A further benefit has been the growth of interest of other professional staff in this system, with the result that they are applying it to their own practice - for example, occupational therapy - and joining with nurses to develop multidisciplinary standards such as that on discharge planning.

\section{Conclusion}

If quality and standards are going to become realities then there has to be a mutually top down and bottom up initiative. Without leadership from the top from those with authority change is more difficult for staff; without the involvement and commitment of practitioners exhortation from the top will sound impressive but achieve little. There is still a need to incorporate other quality activities into this framework and especially to relate them to the quality strategy of the special health authority and its quality directional plan, with its focus on implementing a total quality system. However, we believe that we have achieved a flexible yet coordinated approach which involves nurses from every level and specialty within our special health authority, which encompasses multidisciplinary work, and which we believe will indeed ensure a continuous improvement in nursing.

NHS Management Inquiry. Report. London: Department of Health and Social Security, 1983. (Griffiths report.) 